\title{
Performance of second generation BABAR resistive plate chambers
}

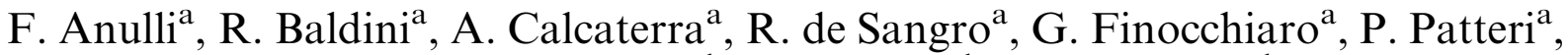
M. Piccolo ${ }^{\mathrm{a}}$, A. Zallo ${ }^{\mathrm{a}}$, C.H. Cheng ${ }^{\mathrm{b}}$, D.J. Lange ${ }^{\mathrm{b}}$, D.M. Wright ${ }^{\mathrm{b}}$, R. Messner ${ }^{\mathrm{c}}$, W.J. Wisniewskic , M. Pappagallo ${ }^{\mathrm{d}}$, M. Andreotti ${ }^{\mathrm{e}}$, D. Bettoni ${ }^{\mathrm{e}}$, R. Calabrese ${ }^{\mathrm{e}}$, G. Cibinetto ${ }^{\mathrm{e}}$, E. Luppi $^{\mathrm{e}}$, M. Negrini ${ }^{\mathrm{e}}$, R. Capra $^{\mathrm{f}}$, R. Contri ${ }^{\mathrm{f}}$, M. LoVetere ${ }^{\mathrm{f}}$,

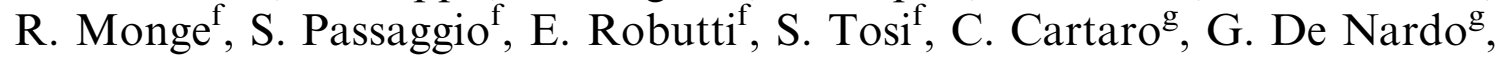
F. Fabozzi ${ }^{\mathrm{g}}$, L. Lista ${ }^{\mathrm{g}}$, D. Monorchio ${ }^{\mathrm{g}}$, D. Piccolo ${ }^{\mathrm{g}}$, P. Paolucci ${ }^{\mathrm{g}}$, R. Covarelli ${ }^{\mathrm{h}}$, M. Pioppi ${ }^{\text {h }}$, M. Carpinelli ${ }^{i}$, F. Forti ${ }^{i}$, N. Neri ${ }^{i}$, E. Paoloni ${ }^{i}$, F. Bellini ${ }^{j}$, G. Cavoto ${ }^{j}$, E. Di Marco ${ }^{j}$, A. D’Orazio ${ }^{j}$, D. del Re ${ }^{j}$, R. Faccini ${ }^{j}$, F. Ferrarotto ${ }^{j}$, F. Ferroni ${ }^{j}$, M. Gaspero ${ }^{j}$, L. Li Gioi ${ }^{j}$, M.A. Mazzoni ${ }^{j}$, S. Morganti ${ }^{j}$, M. Pierini ${ }^{j}$, G. Piredda ${ }^{\mathrm{j}}$, C. Voena ${ }^{\mathrm{j}}$, C. Potter ${ }^{\mathrm{k}}$, N. Sinev ${ }^{\mathrm{k}}$, D. Strom ${ }^{\mathrm{k}}$, S. Foulkes ${ }^{1}$, K. Wang ${ }^{1}$, H.R. Band ${ }^{\mathrm{m}, *}$, J. Hollar ${ }^{\mathrm{m}}$, P. Tan ${ }^{\mathrm{m}}$

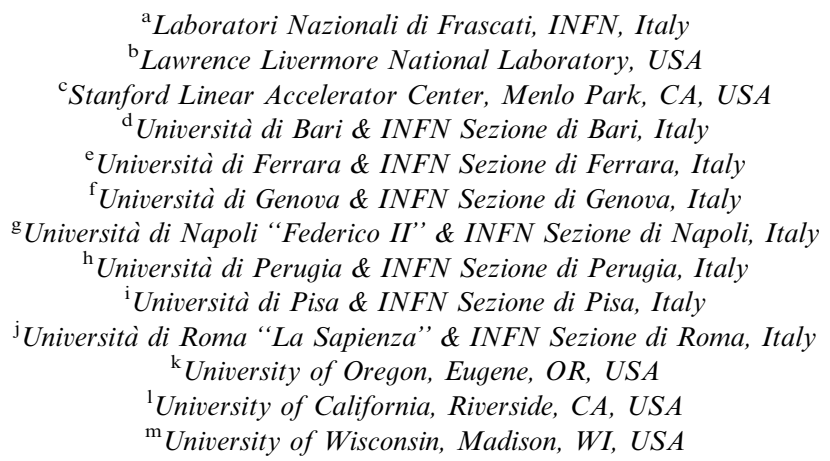

${ }^{a}$ Laboratori Nazionali di Frascati, INFN, Italy

${ }^{\mathrm{b}}$ Lawrence Livermore National Laboratory, USA

${ }^{\mathrm{c}}$ Stanford Linear Accelerator Center, Menlo Park, CA, USA

${ }^{\mathrm{d}}$ Università di Bari \& INFN Sezione di Bari, Italy

${ }^{\mathrm{e}}$ Università di Ferrara \& INFN Sezione di Ferrara, Italy

${ }^{\mathrm{f}}$ Università di Genova \& INFN Sezione di Genova, Italy

${ }^{\mathrm{g}}$ Università di Napoli "Federico II" \& INFN Sezione di Napoli, Italy

${ }^{\mathrm{h}}$ Università di Perugia \& INFN Sezione di Perugia, Italy

${ }^{\mathrm{i}}$ Università di Pisa \& INFN Sezione di Pisa, Italy

${ }^{\mathrm{j} U n i v e r s i t a ̀ ~ d i ~ R o m a ~ " L a ~ S a p i e n z a " ~ \& ~ I N F N ~ S e z i o n e ~ d i ~ R o m a, ~ I t a l y ~}$

${ }^{\mathrm{k}}$ University of Oregon, Eugene, OR, USA

${ }^{1}$ University of California, Riverside, CA, USA

${ }^{\mathrm{m}}$ University of Wisconsin, Madison, WI, USA

${ }^{*}$ Corresponding author. Tel.: + 16509262655 ; fax: + 16509262923.

E-mail address: hrb@slac.stanford.edu (H.R. Band). 


\begin{abstract}
The BABAR detector has operated nearly 200 Resistive Plate Chambers (RPCs), constructed as part of an upgrade of the forward endcap muon detector, for the past two years. The RPCs experience widely different background and luminosity-driven singles rates $\left(0.01-10 \mathrm{~Hz} / \mathrm{cm}^{2}\right)$ depending on position within the endcap. Some regions have integrated over $0.3 \mathrm{C} / \mathrm{cm}^{2}$. RPC efficiency measured with cosmic rays is high and stable. The average efficiency measured with beam is also high. However, a few of the highest rate RPCs have suffered efficiency losses of 5-15\%. Although constructed with improved techniques and minimal use of linseed oil, many of the RPCs, which are operated in streamer mode, have shown increased dark currents and noise rates that are correlated with the direction of the gas flow and the integrated current. Studies of the above aging effects are presented and correlated with detector operating conditions.
\end{abstract}

(C) 2005 Elsevier B.V. All rights reserved.

PACS: $29.40 . \mathrm{C}$

Keywords: Resistive plate chamber; Muon detection; BABAR

\section{Introduction}

The BABAR detector [1], operating at the PEPII B factory of the Stanford Linear Accelerator Center (SLAC), installed over 200 second generation Resistive Plate Chambers [2](RPCs) as part of an upgrade [3] of the forward endcap muon and neutral hadron detector (IFR) in 2002. Most of the RPCs were operated nearly continuously for the one and one half years of BABAR data taking covered in this report. BABAR RPCs operate in limited streamer mode, using a gas mixture of $4.5 \%$ isobutane, $60.6 \%$ argon and $34.9 \%$ Freon 134a. The new RPCs were built from bakelite sheets at General Tecnica [4], the same factory which produced the original BABAR RPCs. A stringent quality assurance (QA) program was developed by the IFR group to keep the inner bakelite surfaces as clean as possible and to ensure that the final linseed oil coating was thin and well cured. New molded corner pieces were designed to replace the drilling method previously used for the gas fittings. Multiple filters were added to the linseed oil filling stations and the oil was periodically analyzed for impurities. Many of the QA improvements in RPC production made by the IFR group were adopted for the production of RPCs used by the LHC collaborations.

The new RPCs have performed reliably, maintaining good efficiency, justifying the design changes that were introduced and the care taken in their construction. However, clear signs of aging are seen in the higher rate chambers. The observed changes in chamber current, noise rate and efficiency are sensitive to the details of the gas flow and may be reversible to some extent. Since the BABAR RPCs share many construction details with the LHC RPCs and will integrate comparable charge per unit area, their aging is of particular relevance to the world-wide RPC detector community.

\section{Detector overview}

The 2002 IFR upgrade [3] replaced RPCs in the forward endcap region and added steel or brass absorber. In addition, the high voltage and gas [5] distribution systems were improved. Elements of the BABAR detector are shown in Fig. 1. There were 17 gaps in the flux return steel filled with RPCs in the original design. As part of the upgrade, new absorber was added to gaps 8,10 , $12,14,16$ and to the outside of the endcap. The remaining interior gaps were filled with a single layer of RPCs. The two new gaps outside the original flux return were each filled with two separate RPC layers. A double layer of RPCs was also added in a belt layer which wraps around the endcap. The RPC layers are numbered from 1 to 16 starting at the layer closest to the interaction point. 


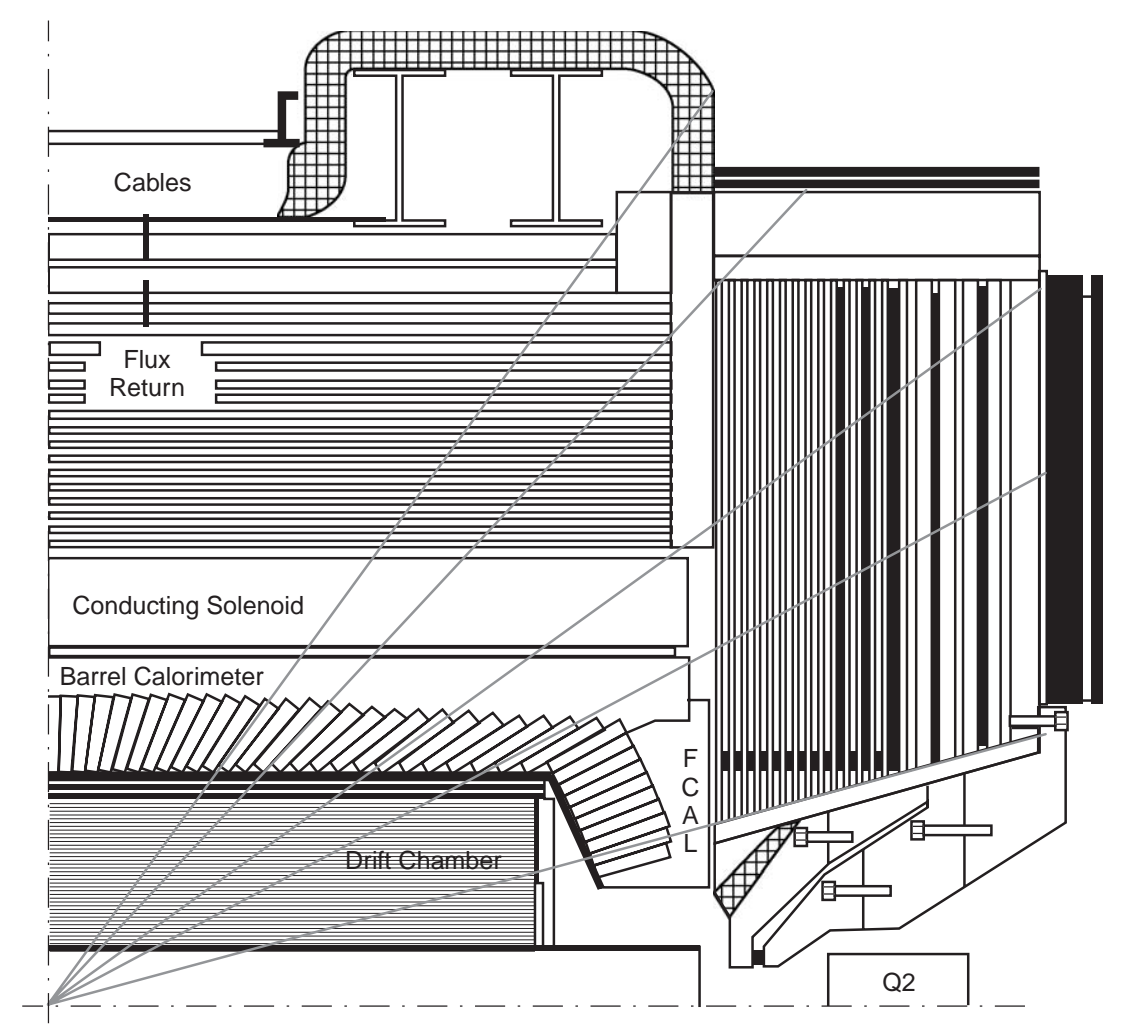

Fig. 1. A longitudinal section of the BABAR detector showing the details of the flux return steel surrounding the inner detector elements. Planar RPCs fill the gaps in the steel. The absorber added in gaps 8,10,12,14,16, and outside the original endcap in 2002 is shown as black. The new endcap contains 16 RPC planes numbered from left to right.

Endcap chambers are made from two RPC highvoltage modules interconnected to form a single gas volume and share one view of the readout strips. The positions of modules within a layer are indicated in the end view of the detector shown in Fig. 2. The modules are numbered from 1 to 6 counting from the bottom. The gas lines of two high-voltage modules are connected in series to form a single gas volume of $\sim 81$. Details of the RPC construction and testing may be found in Ref. [3]. Unless noted otherwise, all measurements and plots in this paper refer to subsets of the RPC modules in Layers 1-16 and do not include the belt chambers.

\section{RPC operating conditions}

All BABAR RPCs use the same gas mixture of $4.5 \%$ isobutane, $60.6 \%$ argon and $34.9 \%$ Freon 134a and operate in the streamer mode. The gas flows in the inner (outer) layers were originally set to $22(45) \mathrm{cc} / \mathrm{min}$ corresponding to $4(8)$ volume changes per day. Concern for increased currents in the higher rate middle chambers prompted an increase in the gas flows in these RPCs to 8 volume changes per day (on day 300). During the Christmas 2003 break (day 420), all flows in the forward endcap were reversed.

A typical efficiency versus high-voltage curve obtained from cosmic ray data is shown in Fig. 3 for two of the RPC modules. The functional form of the efficiency is well described by

$\varepsilon(V)=\frac{\varepsilon_{0}}{e^{\left(V_{0}-V\right) / \Delta_{V}}+1}$.

The fit values for the plateau efficiency $\left(\varepsilon_{0}\right)$, knee $\left(V_{0}\right)$ and width $\left(\Delta_{V}\right)$ are indicated, respectively. At the normal operation voltage of $6700 \mathrm{~V}$ the 


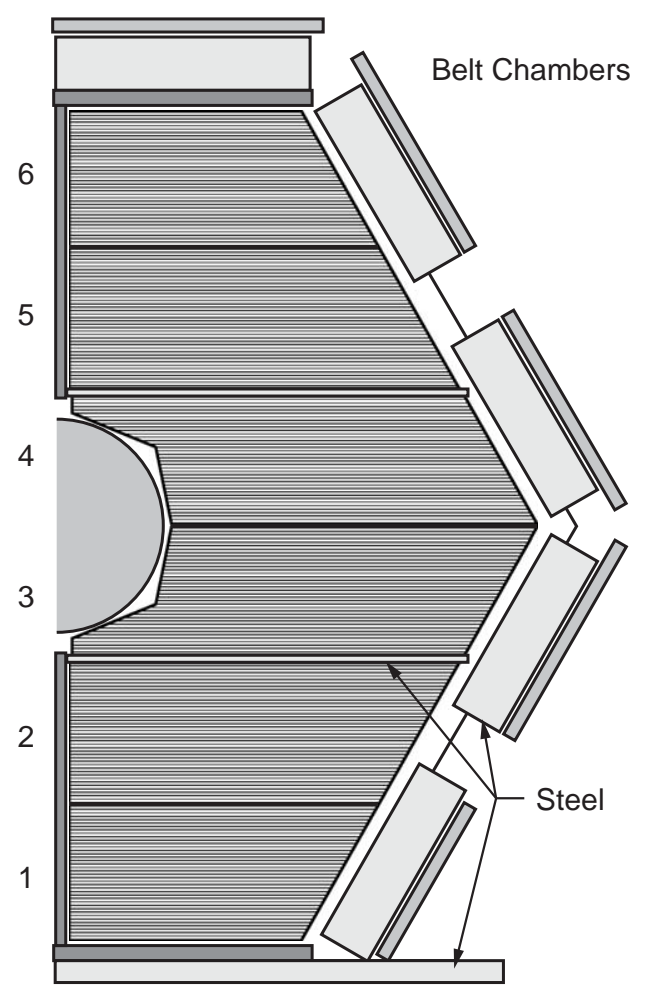

Fig. 2. Typical RPC geometry for a layer in the East door. Each layer contains three chambers. Each chamber is made from two high-voltage modules which share pickup strips and ground planes. In this paper the RPC HV modules will be identified by layer number (1-16) and module number (1-6) counted from the bottom. The gas lines of the two modules $(1-2,3-4,5-6)$ are connected in series. For the first 14 months of operation, the gas entered the bottom module and exited from the upper module.

average charge per streamer is $\sim 1 \mathrm{nC}$. When beams were injected into PEPII the voltage was ramped down to $4500 \mathrm{~V}$, well below the gas gain region. During normal data collection, the RPCs would be at $6700 \mathrm{~V}$ for about $1 \mathrm{~h}$, then ramp down to $4500 \mathrm{~V}$ for 5-10 min while PEPII injected beam, and would then ramp back to $6700 \mathrm{~V}$ to repeat the cycle. PEPII changed injection modes in the spring of 2004 to inject beam continuously, eliminating the need to ramp the voltages. The RPCs were then kept at operating voltages continuously, except for occasional beam down times.

The gas flow, temperature, singles rate and current of the RPCs were continuously monitored

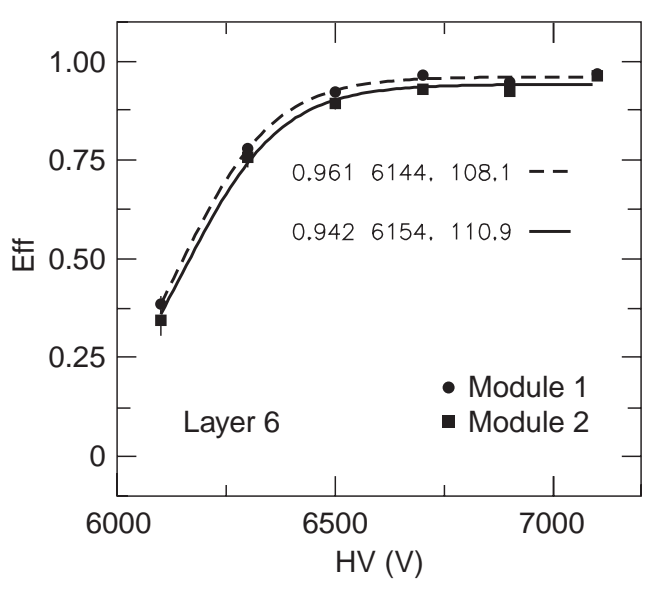

Fig. 3. Efficiency as a function of the RPC voltage for two modules in Layer 6. The data is fit to Eq. (1) obtaining the values of $\varepsilon_{0}, V_{0}$ and $\Delta_{V}$ indicated.

and recorded in the BABAR database. Although the experimental hall was not temperature controlled, the steel of the forward endcap was water cooled, providing a reasonably stable temperature environment for the chambers as shown in Fig. 4. The inner 12 layers were typically at $19-22^{\circ} \mathrm{C}$ while the upper half of the outer four layers was $\sim 2-3^{\circ}$ warmer.

\subsection{Backgrounds}

The streamer rates produced by backgrounds and signals from normal BABAR running varied considerably depending on the layer and position of the chambers. In the inner Layers 1-12 the chamber occupancy was highest around the beam line as seen for Layer 1 in Fig. 5. Signal rates (and occupancy) were proportional to the PEPII luminosity and were typically $30-50 \mathrm{kHz}$ per module in Layer 1 with peak rates above $10 \mathrm{~Hz} /$ $\mathrm{cm}^{2}$. The rates decreased with increasing layer number, reaching a minimum in Layer 11 at $\sim \frac{1}{4}$ of the Layer 1 rate. RPC modules in the bottom of the endcap (position 1) saw very low rates (little more than the cosmic ray rate) and never drew more than a few microamps.

The rates in the outermost Layers (15 and 16) were sensitive to backgrounds from PEPII, which enter the outside of the endcap. These 


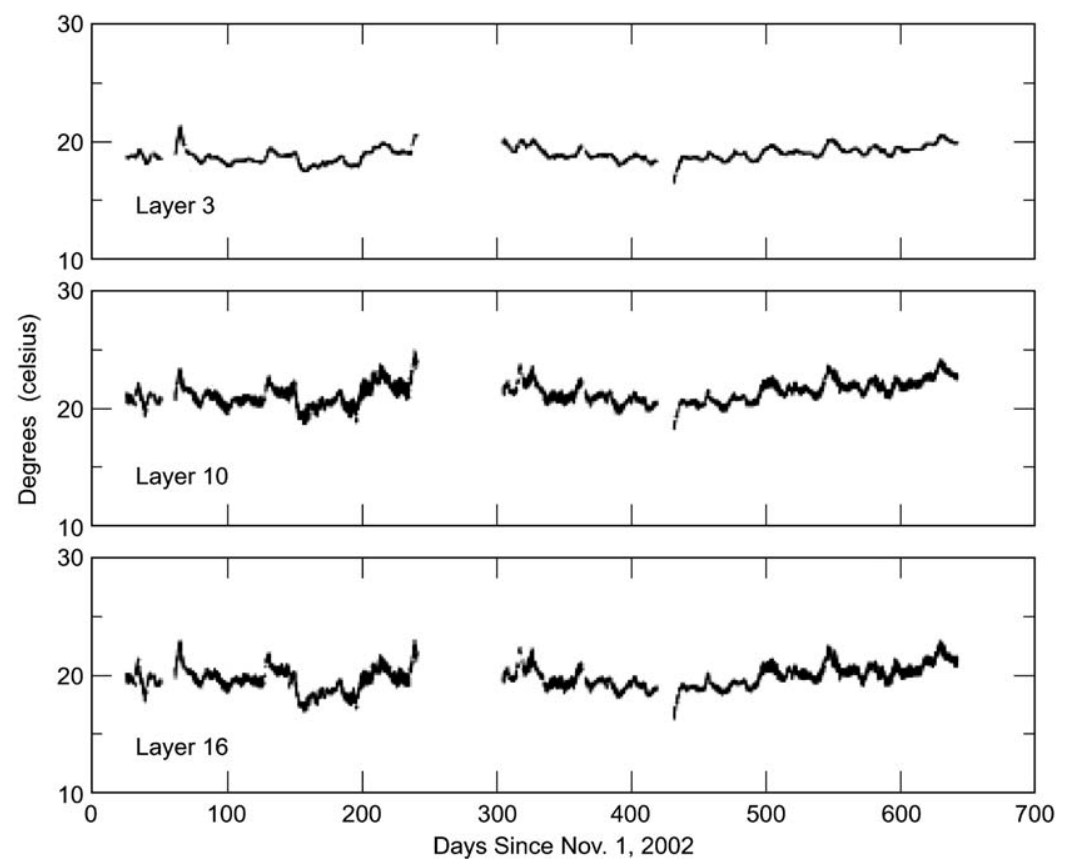

Fig. 4. Average temperature versus time in three layers of the forward west endcap door.

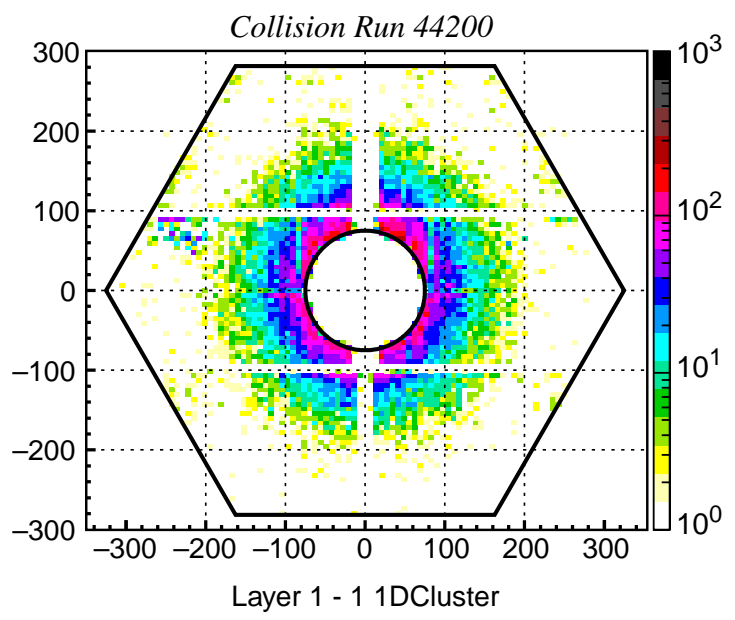

Fig. 5. The RPC occupancy in Layer 1 averaged over $10^{5}$ events during a normal BABAR colliding beam run. A $1 \%$ occupancy corresponds to $10^{3}$ hits.

backgrounds were often too high $\left(\geqslant 12 \mathrm{~Hz} / \mathrm{cm}^{2}\right)$ to allow normal operation. The rates in the next outermost Layers (13-14) were lower $\left(\sim 4 \mathrm{~Hz} / \mathrm{cm}^{2}\right)$ with typical PEPII backgrounds. A two-dimensional hit occupancy map of Layer 14 during a normal collision run is shown in Fig. 6. Although the rates per module of RPCs in Layer 14 were typically higher than for RPCs in Layer 1, hits were spread over much of the chamber surface, resulting in lower peak rates per unit area. Due to the high backgrounds, Layers 15 and 16 were off for most of the data collection. Layer 13 was also kept off as a precautionary measure. A $20 \mathrm{~cm}$ thick steel shielding wall was installed in the summer of 2004 which should allow the operation of all layers in the future.

\section{Performance versus time}

\subsection{Noise rates}

As described in the previous section, the RPC rates were sensitive to luminosity and background conditions. Without beam, the rate was typically orders of magnitude smaller and due either to 


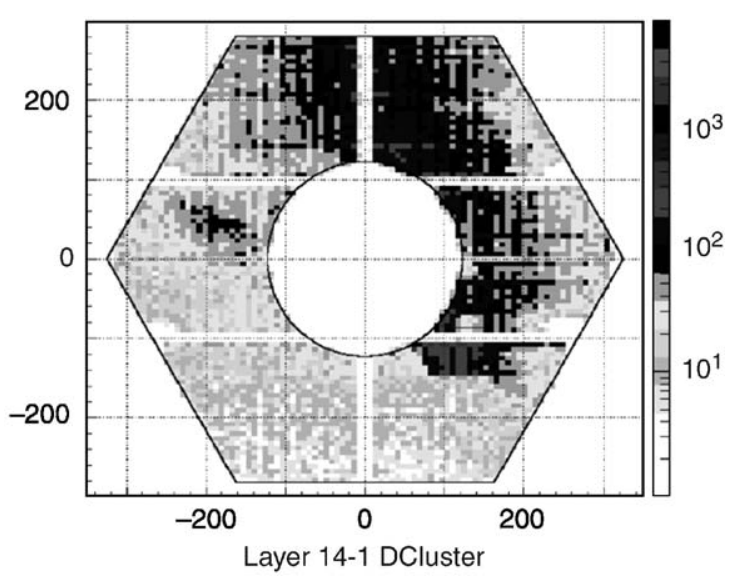

Fig. 6. The RPC occupancy in Layer 14 averaged over $10^{5}$ events during a normal BABAR colliding beam run. Layer 14 is separated by $11 \mathrm{~cm}$ of steel from the experimental hall. The lack of hits near the gas inlets of the middle and top chambers (modules 3 and 5) is evidence for a rate-dependent inefficiency. intrinsic defects in that specific RPC module or to cosmic rays. It was therefore useful to track the rate under cosmic ray conditions as well as with colliding beams. The rates are measured from strips running along the long axis of the RPC module. Whenever a streamer produced a pulse on the strip line $\geqslant 40 \mathrm{mV}$ (streamer signals averaged about $200 \mathrm{mV}$ ), a $100 \mathrm{~ns}$ long TTL pulse was generated. A logical OR was made of the signals from alternate strips. The rate sum of this signal is sampled and recorded every $5 \mathrm{~min}$ by the monitoring electronics. Shown in Fig. 7 are the average rates of the six modules in Layer 14 (west door) as a function of time. The rates with beam vary from $\leqslant 3 \mathrm{kHz}$ to over $300 \mathrm{kHz}$. During the spring of 2004 PEPII/BABAR changed injection modes, trickling in charge continuously with the detectors operating instead of turning off the chambers periodically to inject beam. Surprisingly,

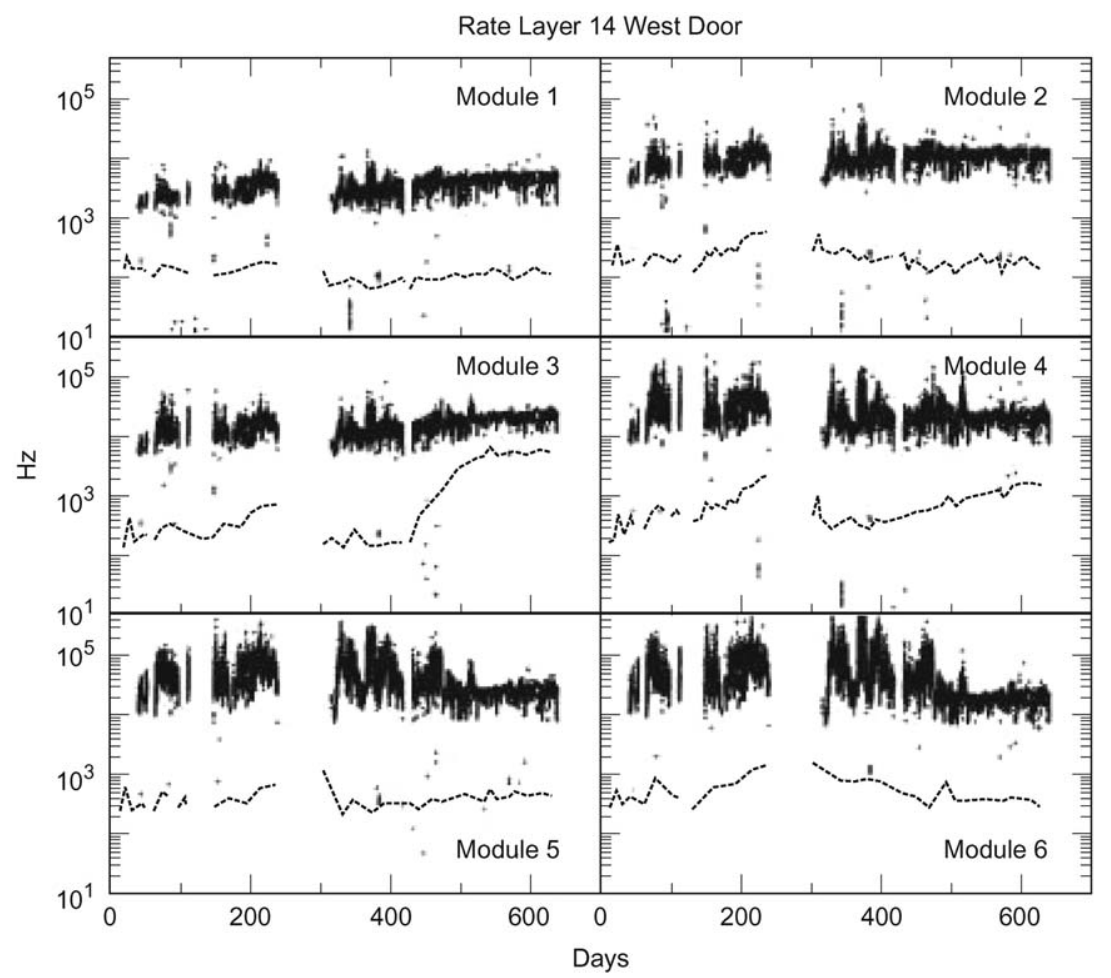

Fig. 7. The average noise rate of the 6 RPC modules in Layer 14 of the West door plotted versus time (days since November 2002). The average noise rate with colliding beams is shown as black points. The average noise rate without beam is shown as a dashed line. 
backgrounds in all the outer layers became more stable and decreased substantially.

The rates without beam often grew slowly with time, particularly in the higher rate modules. This trend can be seen in Fig. 8 which shows the average rate of the 12 inner layers in the west door versus time. The higher rate chambers (positions 3 and 4) show significant increases with time in both Figs. 7 and 8. It is interesting to note that most of the increased rate in modules at positions 1,3 , and 5 occurred after the gas flow reversal at day 420 put them second in the gas chain.

Converting from the measured rate per chamber to a rate per unit area required specialized data and a few assumptions. Random triggers during collision data were recorded to obtain the twodimensional occupancy of coincidences between the horizontal and vertical strips. Figs. 5 and 6 are the resulting distributions when there was a unique $\mathrm{x}-\mathrm{y}$ pair in the chamber. In each inner layer a radial distribution of hits is calculated and scaled by the total number of two-dimensional hits in that layer. The resulting hit density is then multiplied by the total rate of the layer to get the approximate peak rate. The peak rate varies from $5 \mathrm{~Hz} / \mathrm{cm}^{2}$ in Layer $1-1 \mathrm{~Hz} / \mathrm{cm}^{2}$ in Layer 11 . In the outer layers the hit density is assumed to be uniform. The rate for the module is divided by its area to give the rate per unit area. The rates in Layer 14 were typically $3 \mathrm{~Hz} / \mathrm{cm}^{2}$ and occasionally 2-3 times higher.

A study was made to determine the sources of the increased noise rate. Data without beam present were recorded with a random trigger to measure the intrinsic RPC noise. Two-dimensional occupancy maps were then calculated. The occu-

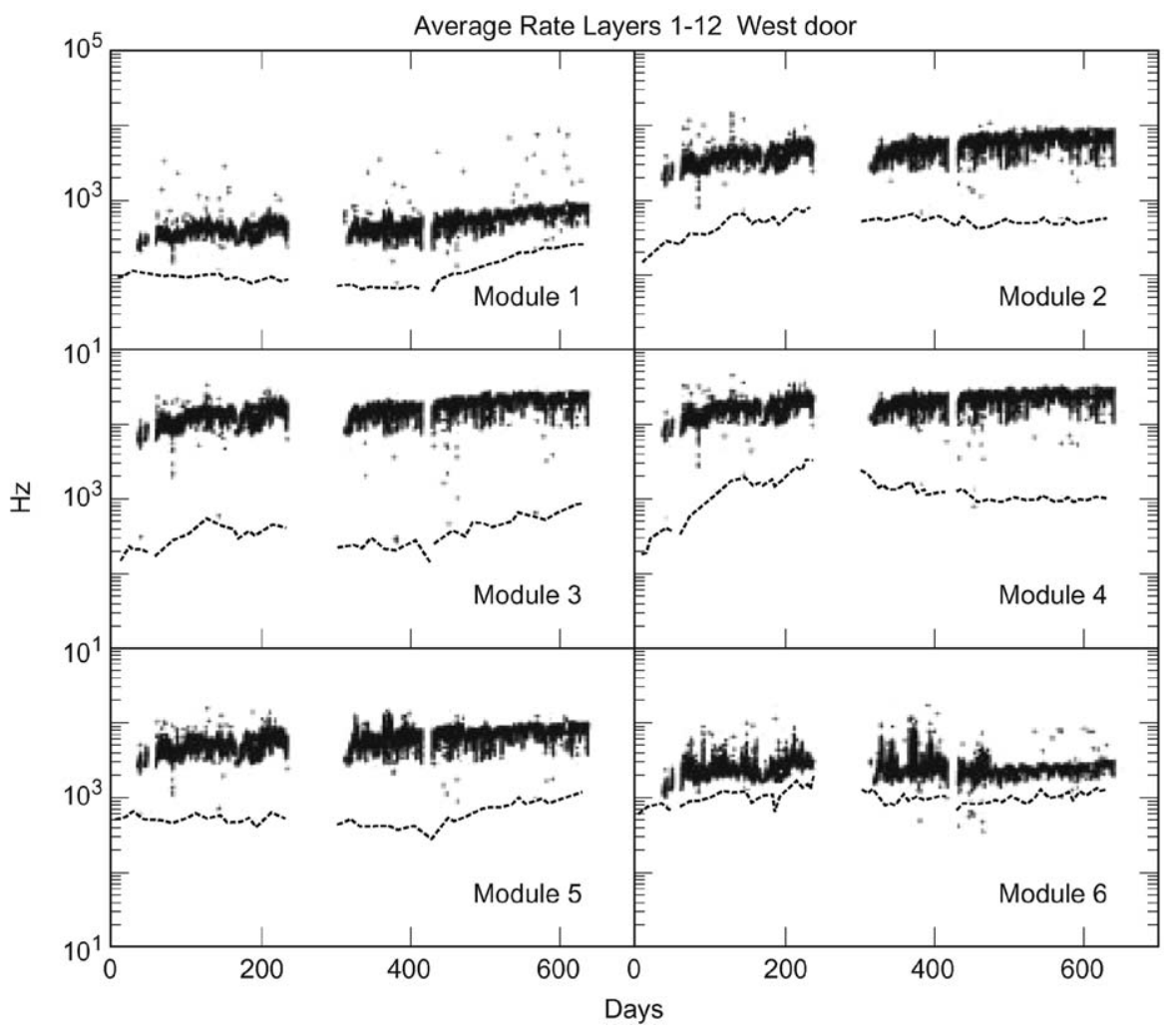

Fig. 8. The average rate of the 12 inner layers for each position plotted versus the days since November 2002 in the west door. The average noise rate with colliding beams is shown as black points. The average rate without beam is shown as a dashed line. 


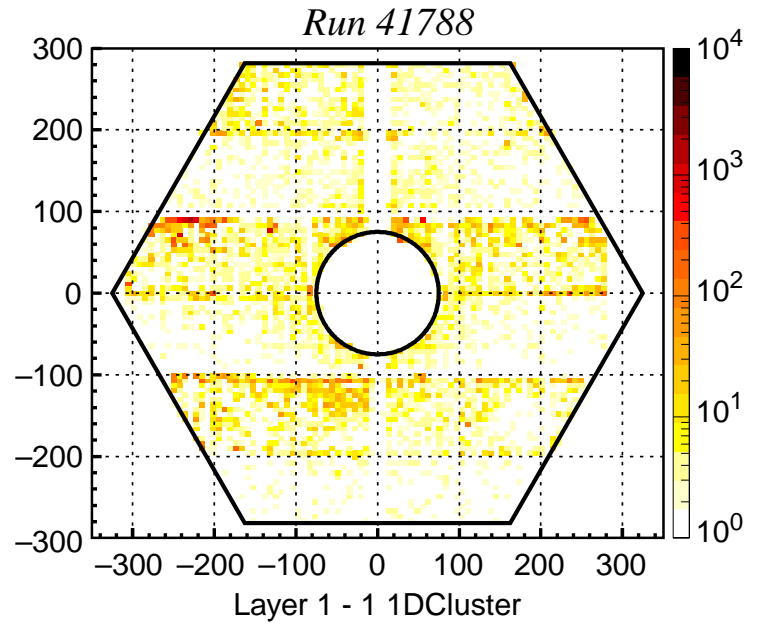

Fig. 9. The RPC occupancy in Layer 1 in data collected with no beam and a random trigger.

pancy map for Layer 1 is shown in Fig. 9 for data collected in the summer of 2003 . The intrinsic chamber noise is not uniform but is concentrated along the edges of the modules and at "hot spots". The upper module of each chamber has a higher noise rate.

Fig. 10 shows the summed occupancy of most of the inner layers. The upper module of each chamber clearly has a higher density of hits than the lower module. In addition, there is a clear increase in number of noise hits in regions of high activity (around the beam-line) with beam. These distributions can be explained in a model where pollutants are produced in the gas proportionate to the noise rate and that the gas flow can move these pollutants downstream.

The evolution in time of RPC rates can be seen in Fig. 11, where the rate of each RPC in Layers 1-11 (four RPCs installed in 2000 are not included) is histogrammed in three time periods: shortly after installation (December 2002), after one year of running (December 2003), and after one and one half years of running (July 2004). The average rate with no beam rises from 340 to $700 \mathrm{~Hz}$ during that time span. Much of the increase is due to the growth of a high-rate tail. In many cases the higher rates were correlated with gas flow problems in specific chambers. Removing

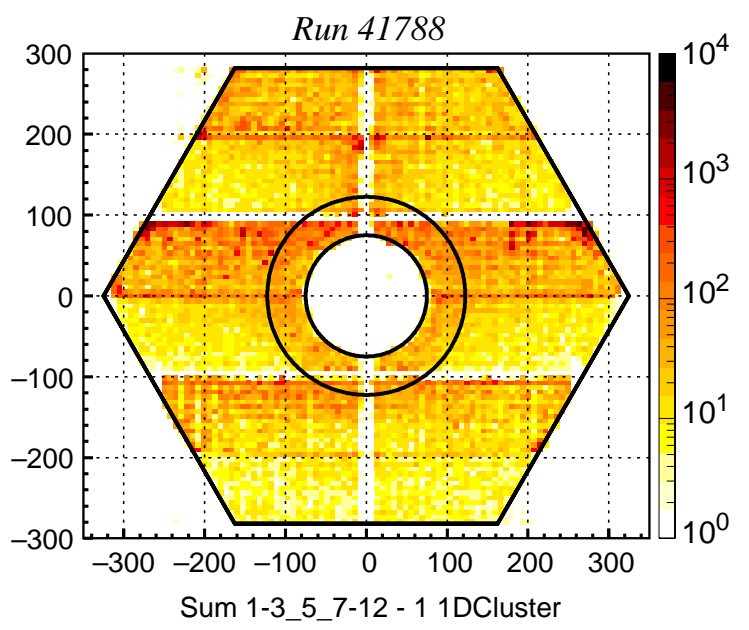

Fig. 10. The RPC occupancy in inner Layers $1-3,5,7-12$ in data collected with no beam and a random trigger.

chambers with known gas flow problems from the latest average reduced the most recent average from 700 to $525 \mathrm{~Hz}$.

\subsection{High-voltage current}

Under normal operation the RPC current is proportional to the hit rate; therefore, the current varies with luminosity or beam background in a similar way to the noise rate. Chambers with increased noise rate also show increased current under cosmic ray conditions. However, some chambers show an additional, larger current increase with time that is not due to real streamers, but is due to the chamber developing a much lower ohmic resistance so that significant current is drawn even without any gas gain. This feature has been estimated by measuring the chamber current at $4500 \mathrm{~V}$, well below the gas gain region and scaling the result to the operating voltage of 6700 V. Shown in Fig. 12 are the current histories of two middle RPC modules in Layer 1. The current from beam-related signals (black points) show similar behavior in both modules. The estimated ohmic current (dashed line) is increasing for the module (b) (second in the gas flow chain). Most of the current increase in time in the second module is due to increases in the ohmic current. 

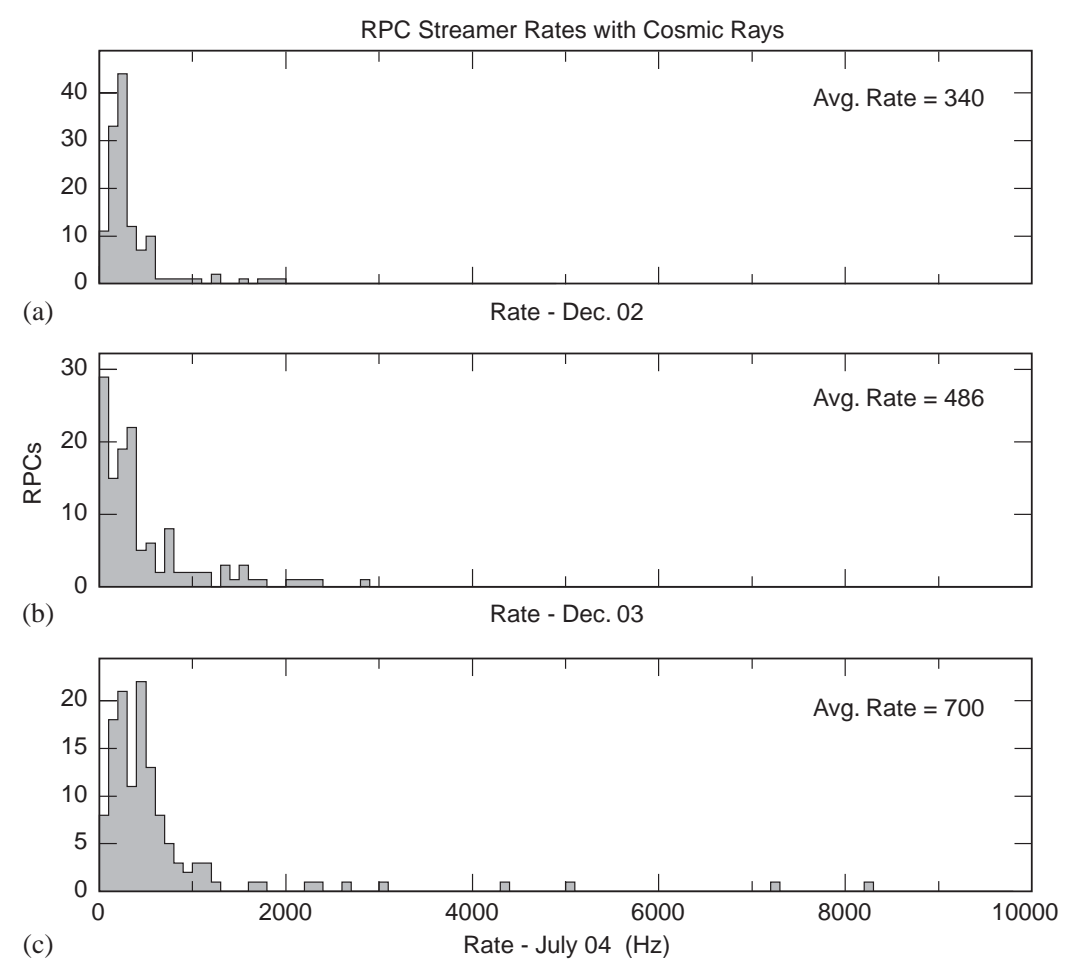

Fig. 11. Distribution of RPC noise rates per HV module in (a) December 2002, (b) December 2003, and (c) July 2004. Both a widening of the low-rate noise peak and the growth of a higher rate tail are evident. Most of the RPCs with high rate suffered from gas flow problems.

This effect can also be seen with the chamber current measured with only cosmic rays present. In this case the ohmic current nearly saturates the measured current. The largest increases in the ohmic current were associated with RPCs which accumulated the most charge and were the second module in the gas flow string.

By averaging over the inner layers as before, the finer details of the dependence are revealed as shown in Fig. 13. Worrisome increases were seen in currents of the modules in position 4. The current increases were slowed by increasing the gas flow after the summer of 2003. Reversing the gas flow in December 2003 significantly reduced the ohmic currents in the previously downstream chambers $(2,4,6)$. Small, but measurable increases in the ohmic current were seen in the previously upstream chambers $(1,3,5)$ after the gas flow was reversed.

The distribution in RPC currents with cosmic rays was also compared at several different times as seen in Fig. 14. Initially (a), the average current was less than $1 \mu \mathrm{A} /$ chamber. This grew to $4.6 \mu \mathrm{A}$ after one year (b) with several chambers having currents exceeding $20 \mu \mathrm{A}$. Improvements to the gas flow reduced the higher currents, slightly reducing the overall average during the next six months (c).

\subsection{Efficiency}

The average RPC efficiencies per module were measured using muon pairs produced during colliding beam data or using cosmic rays. The two methods provide different measures of the average module efficiency. Cosmic ray tracks uniformly illuminate the entire RPC, whereas the muon pair tracks preferentially populate the smallest polar angles. The overall module rate is 10-100 times higher during collision data. The average efficiency of the inner 12 layers measured by cosmic rays and muon pair events is shown in Fig. 15. The efficiency measured with 


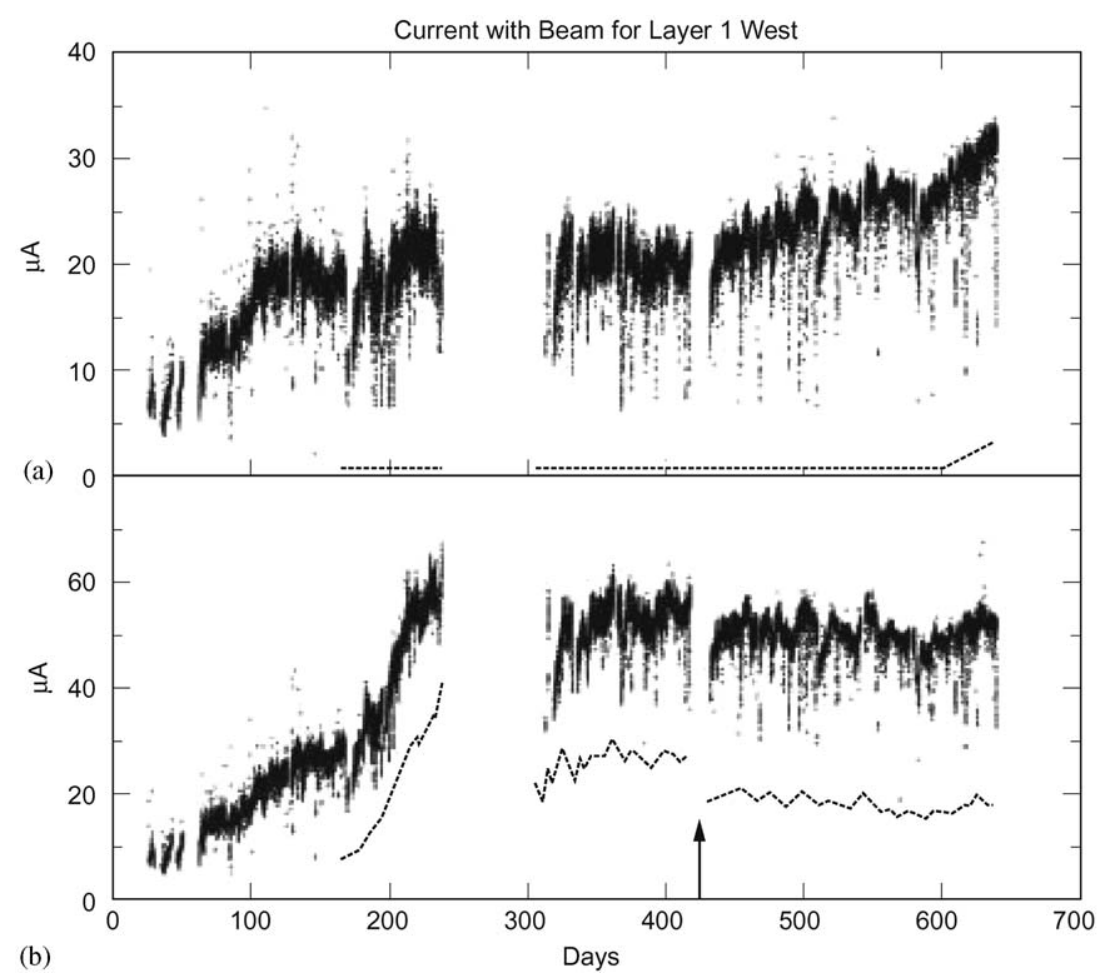

Fig. 12. The current in $\mu \mathrm{A}$ drawn by two RPCs in Layer 1 west near the detector mid-plane. The gas entered the bottom chamber (a) and exited the top chamber (b) until the gas flows were reversed in December 2003 (arrow). The current with beam (black points) has contributions from streamers and an ohmic contribution (dashed line) which is estimated from the current when the voltage was below the gas gain region $(4500 \mathrm{~V})$.

cosmic rays is nearly flat. The efficiency measured with muon pairs was flat during the first data taking period. After the summer shutdown of 2003 the average efficiency was $2 \%$ higher, but with a significant downward trend which was interrupted by the gas flow reversal. Although the average efficiency was unchanged by the end of the run (July 2004), some chambers (crosses) suffered a loss of efficiency as seen in Fig. 15. A closer look at the measured efficiencies with beam shows that the average efficiency trends are different for RPCs in different positions. The average efficiencies for Layers $1-11$ are plotted by position for the east door in Fig. 16. The efficiency of modules in positions $1,2,5$, and 6 were stable with time. The efficiency of both high-rate middle chambers (3 and 4) decreased with time. The faster efficiency decline of modules in position 3 was stopped by the gas flow reversal previously discussed. The slower efficiency decline of modules in position 4 continues.

A summary of the RPC efficiency changes with beam is shown in Fig. 17, which shows the distribution of efficiencies at installation, after one year of running, and after one and one half years of operation. Although the average efficiency is unchanged, two different effects are balanced in the average. First, the efficiency of the lower rate chambers has slightly increased in time. Second, a lower efficiency tail has developed for the higher rate middle chambers.

Using a large sample of muon pairs the efficiency of each chamber was mapped in two dimensions. Such a map for Layer 2, measured in July 2004, is shown in Fig. 18. The ring around the central beam-line has lower efficiency and corresponds to the high-rate region. Smaller inefficient regions are located near the gas inlets. The 

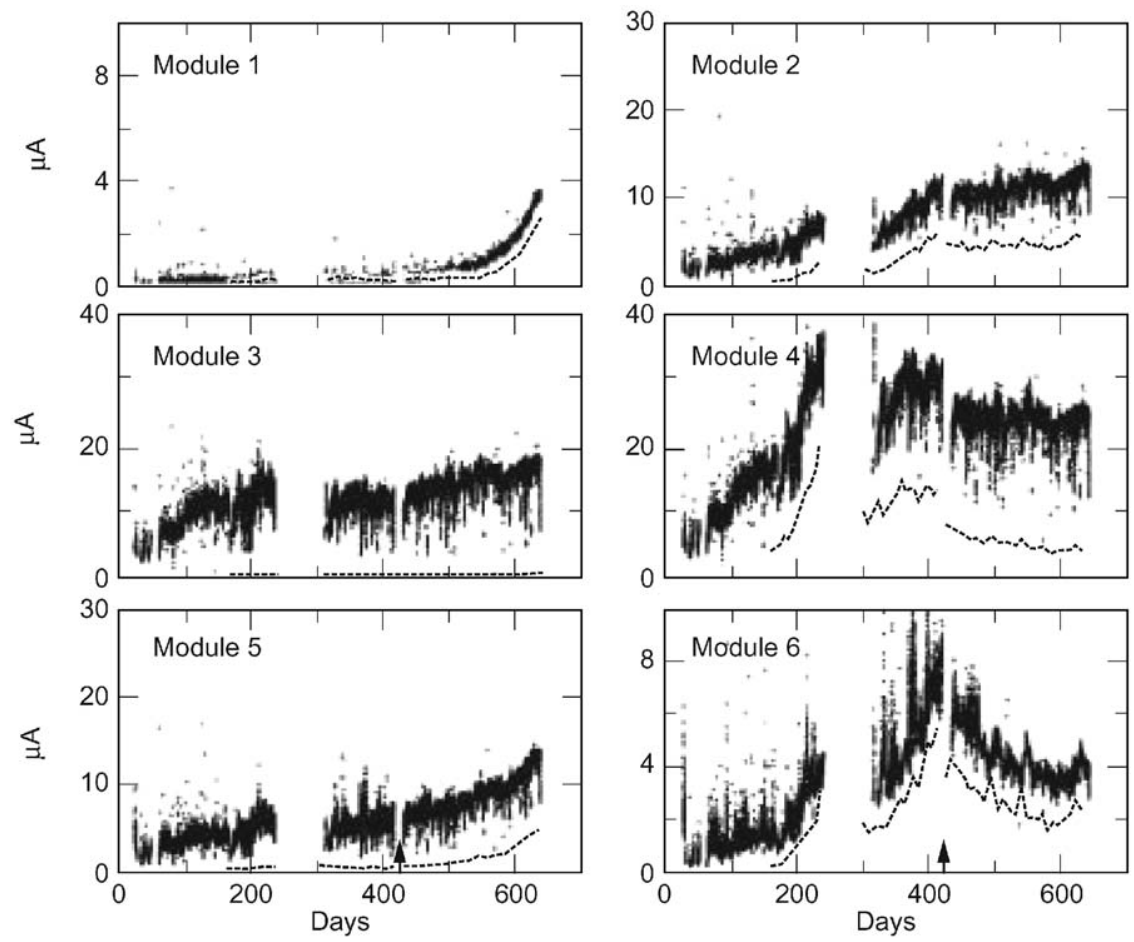

Fig. 13. The average current drawn by Layers $1-12$ in the west door as a function of time. The gas flow was increased in all middle chambers ( 3 and 4 ) on day 280. The gas flows were reversed on day 425 (arrow). The current with beam (black points) has contributions from streamers and an ohmic contribution (dashed line) which is estimated from the current when the voltage was below the gas gain region $(4500 \mathrm{~V})$.

distributions are what one would expect from a rate-dependent inefficiency. The corresponding map of Layer 14 is shown in Fig. 19. The inefficient regions near the gas inlets are larger. Completely missing is the inefficient region around the beam-line. Since the noise rates in Layer 14 are much more uniform than in Layer 1, both observations are consistent with a rate-dependent inefficiency hypothesis.

\subsection{Correlations}

Correlations between the RPC parameters were investigated to provide more information on the chamber performance. Plotted in Fig. 20 is the RPC efficiency with beam as a function of the RPC current in December 2003. The three groups plotted show different behaviors. The lower rate modules $(1,2,5$, and 6) have the lowest currents and highest efficiencies. Modules in position 4 have the largest currents but still have efficiencies above 90\%. Modules in position 3 have the lowest efficiencies despite moderate currents. The roughly linear dependence of the efficiency on the current is consistent with the bakelite resistance limiting the efficiency. The steeper slope of the module 3 chambers implies that these modules have a higher resistance than the module 4 chambers. The clear separation of the module 3 and 4 trends disappeared after the gas flows were reversed. By July 2004 (Fig. 21) the difference between the two slopes lessened and the overlap between the two distributions had increased.

\section{Discussion}

\subsection{Efficiency trends}

As described in the previous sections, most of the endcap RPCs have stable efficiencies with 

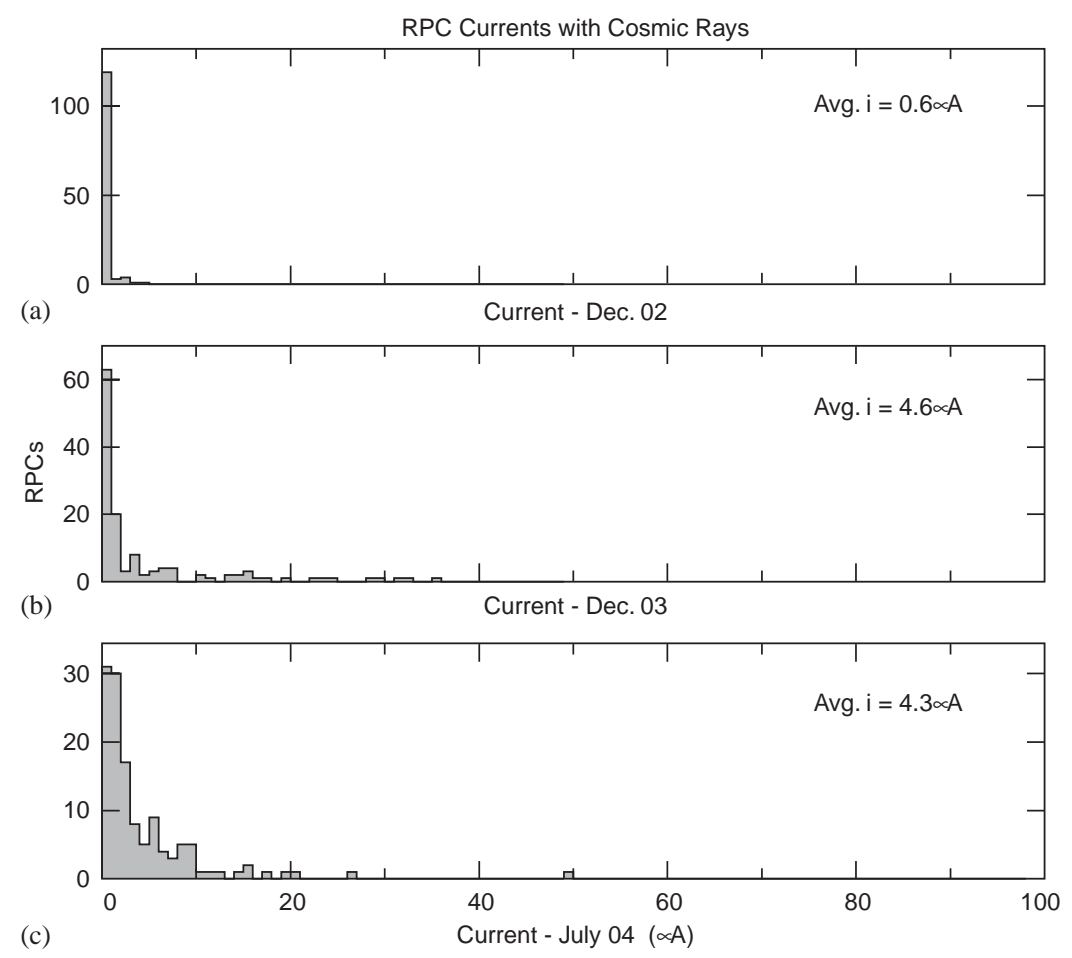

Fig. 14. Distribution of RPC currents measured with cosmic ray backgrounds in (a) December 2002, (b) December 2003, and (c) July 2004. Increased gas flow and the reversal of gas flow direction reduced the currents in several high current chambers.

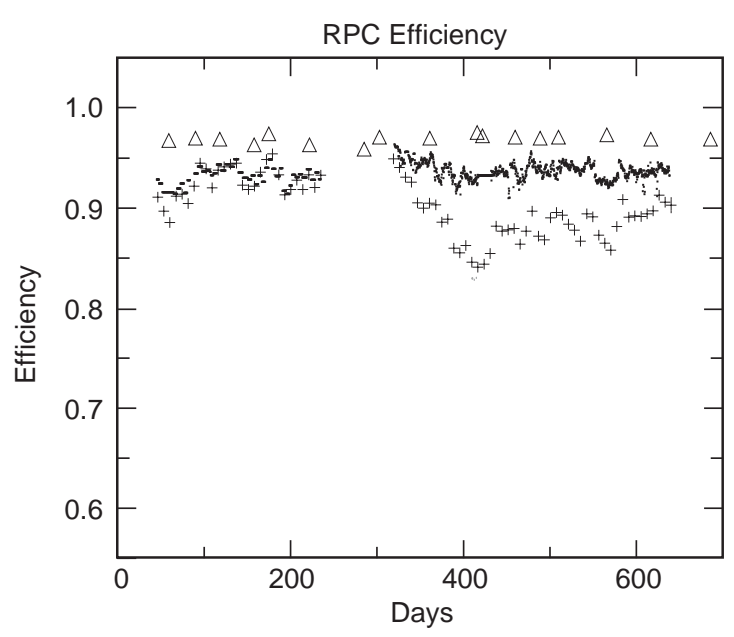

Fig. 15. The average RPC efficiency of Layers $1-12$ was measured with beam using $\mu$-pair (black points) and with cosmic rays (open triangles). The crosses show the efficiency of the fourth RPC in layer 1 of the west door. moderately increasing currents and noise rates. Other RPCs have significantly increased noise rates and currents coupled with significant efficiency losses. Among the second group are eight chambers which had gas flow problems caused by a broken gas connectors. Removing these modules from the sample reduced the number of high noise rate or high current outliers. The remaining RPCs showed a clear correlation between the streamer rate or current and the efficiency loss.

At least part of the observed efficiency changes are probably due to increases of the bakelite resistance with time (due to the bakelite drying out) as has been previously observed by the ATLAS experiment during LHC chamber testing [6]. Initial measurements indicate that the relative humidity of the IFR input gas is $\sim 0 \% \mathrm{RH}$, while the exhaust gas from 2 modules in series is $20-30 \% \mathrm{RH}$, consistent with the removal of 


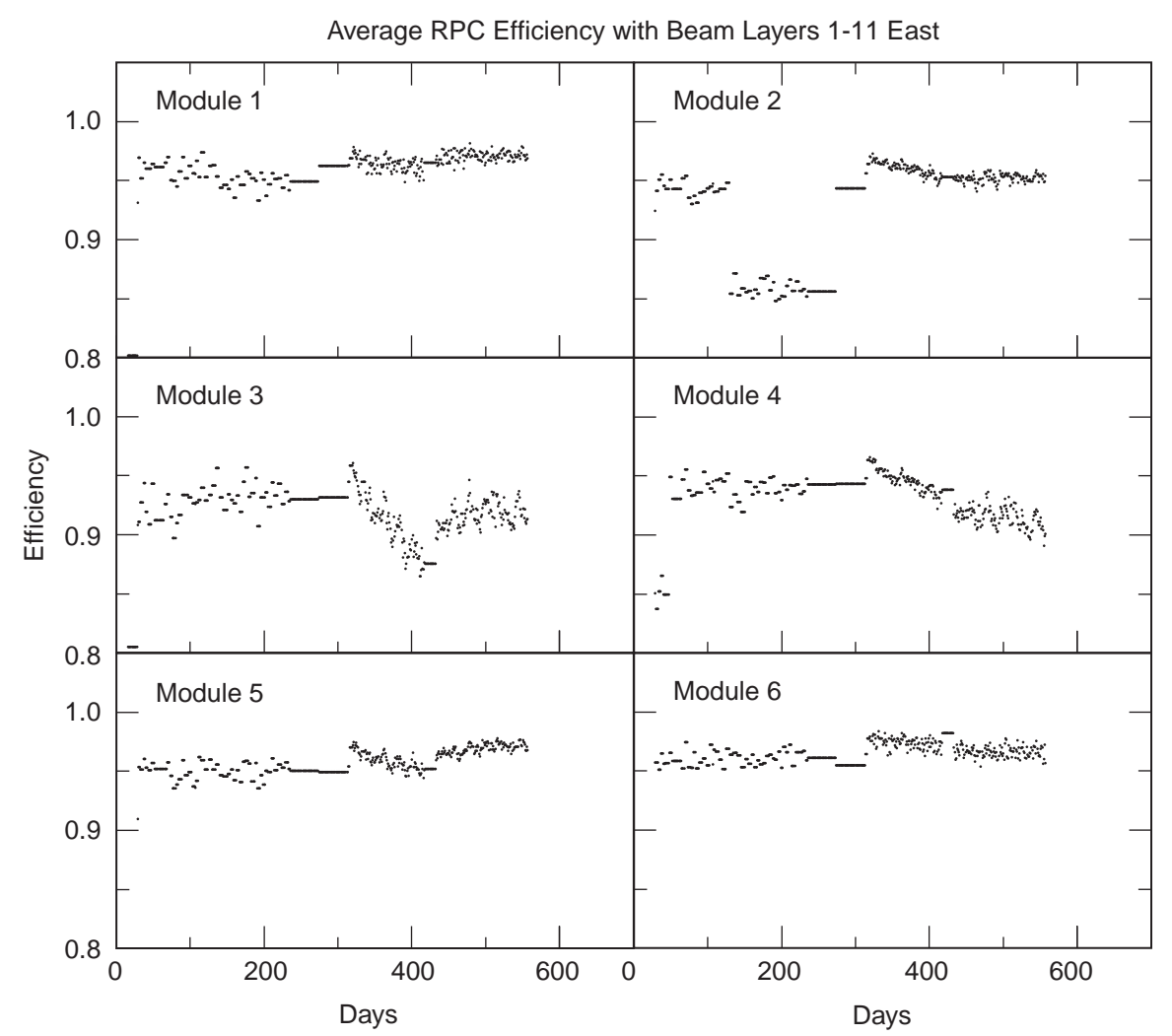

Fig. 16. The average RPC efficiency of Layers 1-11 measured with beam using $\mu$-pairs. The step-like drop and rise of the position 2 efficiency were due to temporarily turning off a chamber due to poor gas flow.

water from the RPCs. This observation suggests the following model. The initially dry gas entering the first module absorbs water from the bakelite as it flows through the module. The removal of water from the bakelite raises the bulk resistivity. When exposed to substantial signal rates, the higher resistance leads to a voltage drop across the bakelite, reducing the voltage across the gap thus lowering the RPC efficiency.

The regions near the gas inlets are exposed to the driest gas at high flows. There is strong evidence in BABAR of high resistance regions developing in the bakelite near the gas inlets. These regions have a much reduced observed noise rate (Fig. 6) or reduced efficiency (Figs. 18 and 19). When the gas flows were reversed and gas inlets became outlets, thereby increasing the humidity of the gas in that regions, the efficiency returned to normal. New inefficient regions appeared at the new gas inlet locations.

It is likely that most of the water removed in the exhaust gas is from the first module. Preliminary measurements show that the humidity of the gas from the BABAR belt chambers (about $\frac{1}{4}$ the size of the typical layer $1-16$ chambers) is already $80 \%$ of that measured in the larger chambers. The gas entering the second (downstream) module is therefore more humid and drying effects in the second module are reduced. This may explain why the gas flow reversals also affected the efficiency in the region of the high-rate ring around the beamline which is far from the gas inlet/outlets. The efficiencies of the chambers originally first in the gas flow chain increased after the reversal (Fig. 16). A definitive test of the above model is 

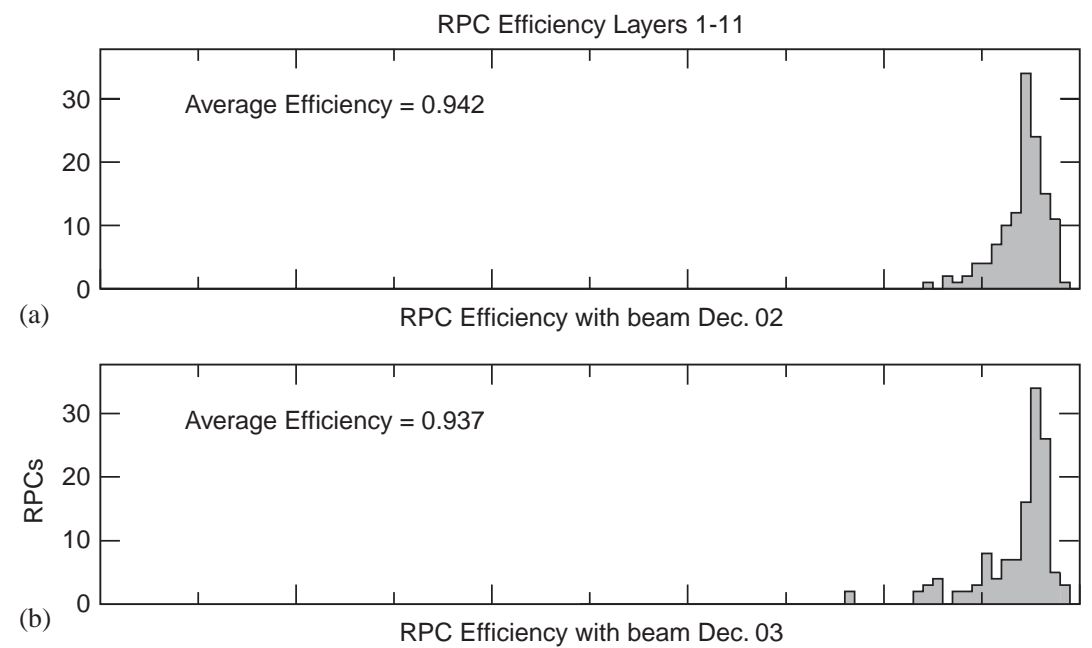

(c)

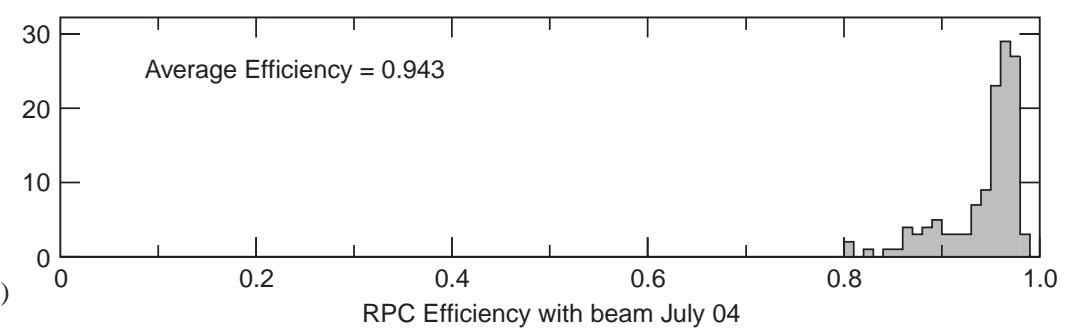

Fig. 17. The distribution of RPC efficiencies in Layers 1-11 measured with beam using $\mu$-pairs in (a) December 2002, (b) December 2003, and (c) July 2004.

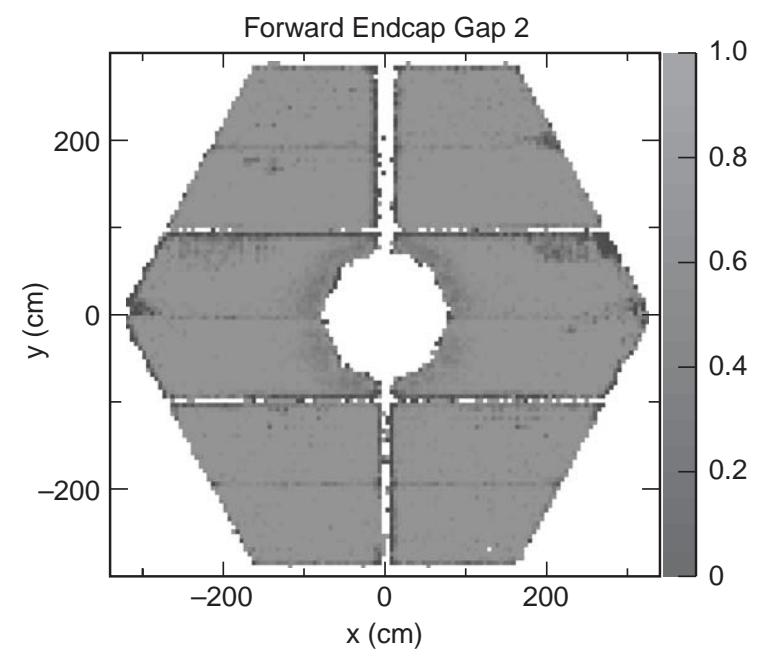

Fig. 18. The two-dimensional RPC efficiency of Layer 2 measured with beam using $\mu$-pairs in July 2004. Lower efficiency regions near the gas inlets are visible as well as a ring of reduced efficiency around the beamline. scheduled for the next run when humidified gas will be sent to a sub-sample of the forward RPCs.

\subsection{Increased noise rates and currents}

The correlation between increased dark currents, background rates, and position in the gas flow string suggests that the downstream RPCs are being exposed to contaminants in the gas produced in the first RPC. Several RPC tests [7] have independently measured the presence of hydrogen fluoride (HF) in the exhaust gas presumably from the decomposition of the freon in the avalanche or streamer. High HF levels have been also correlated to increased ohmic currents [8]. Applying the same model to the BABAR chambers would explain the preferential rise of "hot spots" in the high-rate regions and in the downstream regions. The implication is that aging effects would be reduced by increased gas flow. 


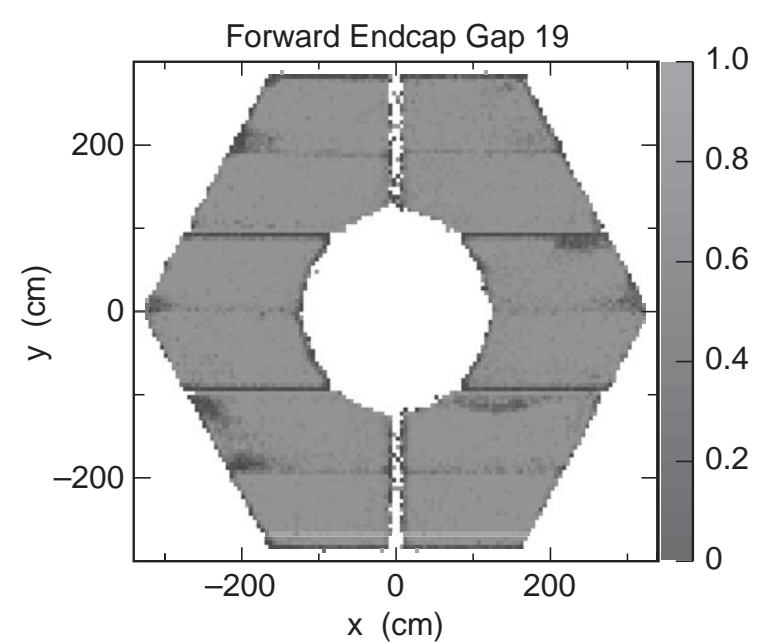

Fig. 19. The two-dimensional RPC efficiency of Layer 14 measured with beam using $\mu$-pairs in the July 2004. Lower efficiency regions near the gas inlets are visible.

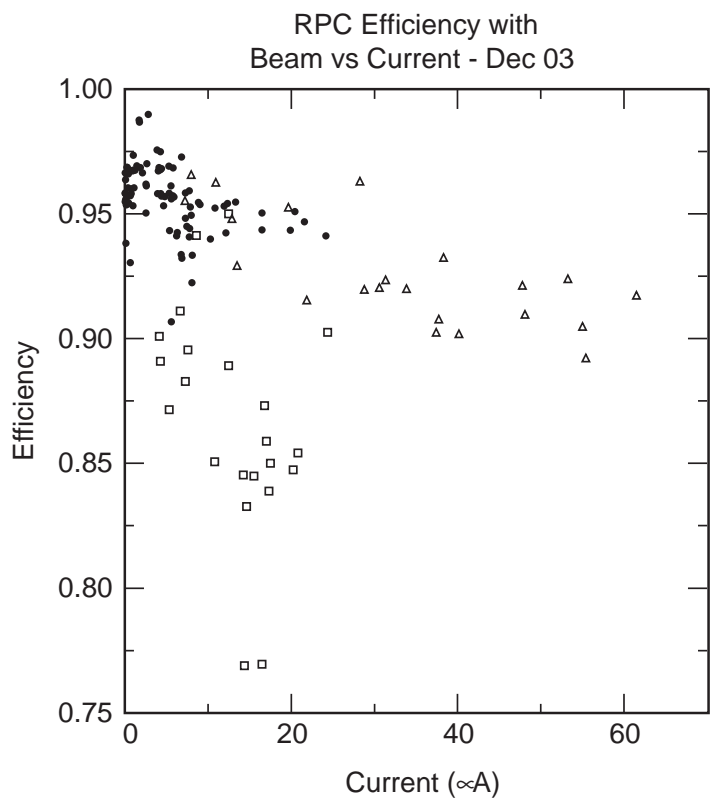

Fig. 20. RPC efficiency in December 2003 plotted against the current (both measured with beam) of modules in Layers 1-11. The solid circles are modules in positions $1,2,5$, and 6 . The open squares (triangles) are modules in position 3 (4).

\subsection{Integrated charge}

The 2002 RPCs in the forward endcap have integrated very different amounts of charge

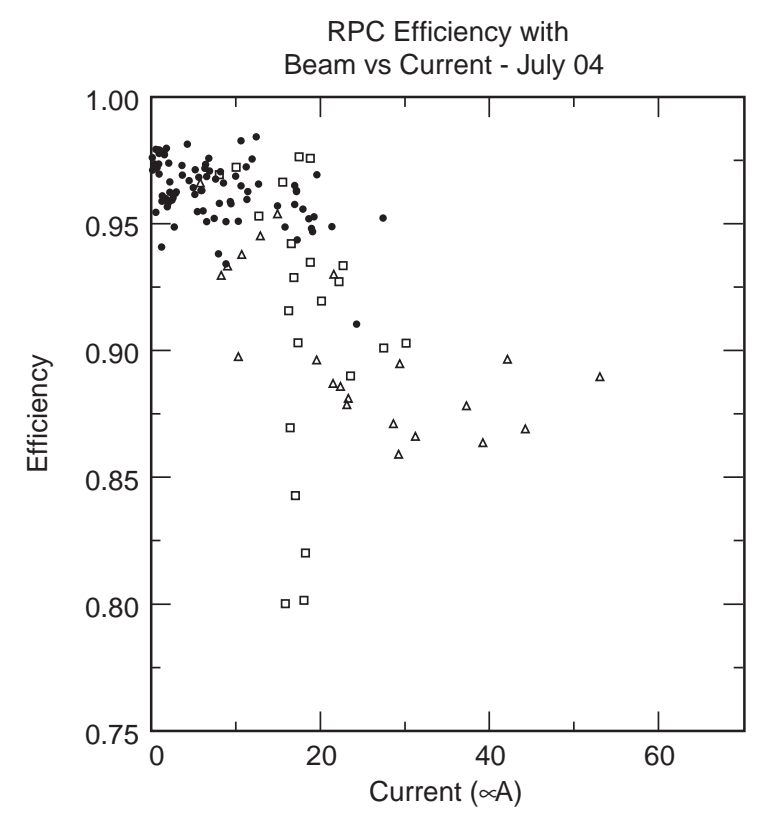

Fig. 21. RPC efficiency in July 2004 plotted against the current (both measured with beam) of modules in Layers 1-11. The solid circles are modules in positions $1,2,5$, and 6 . The open squares (triangles) are modules in position 3 (4).

depending on position and background rates. By integrating (at all voltages) the current history for each high-voltage module, an integrated charge for each module was computed as shown in Fig. 22. The total integrated charges vary from 10 to 1600 Coulombs. The modules which have integrated more than 1000 Coulombs are either in the outermost active layer (14) or in the middle chambers of the inner layers. As seen in Figs. 5 and 6 the distribution of hits is quite different for these two cases. In Layer 14, the hits are fairly uniformly distributed. Assuming an uniform current distribution the integrated charge per unit area can be estimated to be $\approx 0.075 \mathrm{C} / \mathrm{cm}^{2}$. For the inner layers, the current is assumed to be distributed as the two-dimensional hit occupancy (Fig. 5) leading to an estimated peak integrated charge of $\sim 0.3 \mathrm{C} / \mathrm{cm}^{2}$. Despite these high values, no dependence of the RPC efficiency on integrated charge at the module level is seen. 


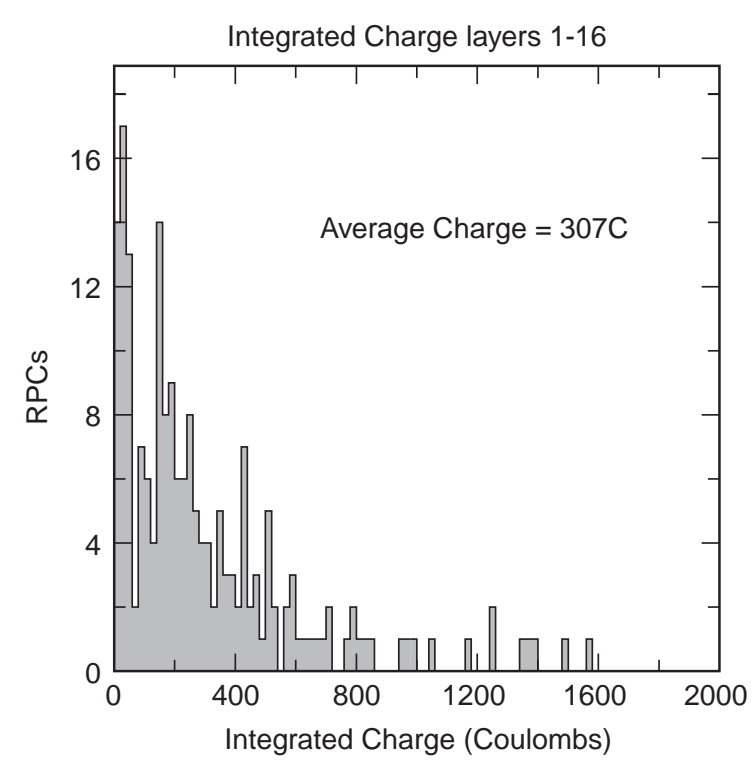

Fig. 22. Total integrated charge of forward endcap modules from November 2002 to July 2004. The modules which vary in size from 1.6 to $2.1 \mathrm{~m}^{2}$ have integrated from 10 to nearly 1600 Coulombs.

\section{Conclusions}

The operational parameters of the second generation BABAR RPCs continue to evolve in response to the observed changes in current, noise rate, and efficiency. Since the BABAR experimental program is expected to extend to 2008 or beyond, maintaining the present high efficiency is of the utmost importance. The overall performance of the new chambers is much superior to the original BABAR RPCs, justifying the design changes and care of construction. However, clear aging effects are seen in the highest rate chambers. Studies are underway which will show if these changes can be controlled by humidifying the input gas. Measurements of the HF concentration in the exhaust gas may lead to further optimization of running parameters.

\section{Acknowledgments}

We wish to thank our PEPII colleagues for their dedicated efforts to constantly improve luminosity and reduce detector backgrounds. This work was supported by the US Department of Energy and the I.N.F.N in Italy.

\section{References}

[1] B. Aubert, et al., Nucl. Instr. and Meth. A 479 (2002) 1.

[2] R. Santonico, R. Cardarelli, Nucl. Instr. and Meth. A 187 (1981) 377.

[3] F. Anulli, et al., Nucl. Instr. and Meth. A 539 (2005) 155.

[4] General Tecnica S. r. 1., I-03030 Colli (FR), Italy.

[5] S. Foulkes, et al., Nucl. Instr. and Meth. A 538 (2005) 801.

[6] G. Aielli, et al., Nucl. Instr. and Meth. A 533 (2004) 86.

[7] R. Santonico, Nucl. Instr. and Meth. A 533 (2004) 1.

[8] G. Aielli, et al., " $\mathrm{F}^{-}$ions production in RPC operated with fluorine compound gases", Talk at IEEE 2004, Roma, Italy (unpublished). 